\begin{abstract}
Aim: Our aim was to develop a method of migration analysis using the undisturbed microcirculation of rat mesentery, and using the new method, analyze leukocyte migration in casein-induced inflammation. Method: Sprague Dawley (SD) rats were injected with tumor necrosis factor (TNF) $\alpha$, interleukin (IL)-1 $\alpha$, or casein intraperitoneally. Following this, the rats were sacrificed and the mesentery tissue removed was fixed and stained with Giemsa. The leukocytes were counted as a rolling index in the venules and as a migration index in the perivascular area.

Results: There was no relation between the diameter of venules and leukocyte migration. The time change curves of leukocyte activity in casein inflammation show about a $1 \mathrm{~h}$ difference between rolling and migration. From inhibitor experiments of caseininduced migration at $2 \mathrm{~h}$, it has been suggested that selectin-related rolling is necessary. Platelet-activating factor (PAF) also appears partially involved.

Conclusion: The improved undisturbed microcirculation method is helpful not only for rolling analysis but also in analysis of leukocyte migration. Casein inflammation analyzed using this method revealed that rolling is necessary and also suggested that partial involvement of PAF is necessary for pathogenesis of leukocyte extravasations.
\end{abstract}

Key words: The undisturbed microcirculation of the rat mesentery, migration, rolling, fucoidin, platelet-activating factor, Tumor necrosis factor- $\alpha$, interleukin- $1 \alpha$, Casein inflammation

\section{Analysis of leukocyte rolling and migration - using inhibitors in the undisturbed microcirculation of the rat mesentery - on inflammatory stimulation}

\author{
Yuko Takano-Ishikawa ${ }^{1}$, Masao Goto ${ }^{1}$ and \\ Kohji Yamaki ${ }^{2, C A}$
}

${ }^{1}$ National Food Research Institute, Kannondai, 2-1-12, Tsukuba, Ibaraki 305-8642, Japan and ${ }^{2}$ National Agricultural Research Center for Tohoku Region, National Agricultural Research Organization, Arai Fukushima 960-2156, Japan

\author{
${ }^{\mathrm{CA}}$ Corresponding author \\ FAX: + 81-24-538-2155 \\ E-mail: kyamaki@affrc.go.jp
}

\section{Introduction}

It is well known that inflammatory recruitment of leukocytes is a multi-step process, involving initial margination in the vessels, rolling and firm adhesion of leukocytes to the vessel wall, and extravasation. The rolling step is very important for leukocyte migration, as it is a prerequisite for leukocyte extravasation or lymphocyte homing. Leukocyte rolling on the vessel wall is mediated by members of the selectin family, which are lectin-like $\mathrm{Ca}^{2+}$-dependent carbohydrate-binding proteins. ${ }^{1}$ L-selectin (CD62L) is constitutively expressed on lymphocytes, and $\mathrm{P}$ selectin (CD62P) and inducible E-selectin (CD62E) are expressed on endothelial cells. ${ }^{1,2}$ P-selectin is stored in an inactive form in $\alpha$-granules of platelets and in the Weibel-Palade body of endothelial cells. ${ }^{2}$ In order to study the rolling, adhering and extravasation of leukocytes in detail, dynamic analysis of leukocyte rolling in rat mesentery venules by intravital microscopy is very useful. However, this method has some shortcomings, including the occurrence of trauma-induced leukocyte rolling and the difficulty in conducting long-term experiments without disturbances resulting from the surgical procedures em- ployed. We have used a combination of methods, including intravital microscopy and histological quantitation of intravascular leukocyte concentration, in the analysis of histamine-induced leukocyte rolling in rat mesentery venules. Especially the histological method is a good method under "undisturbed" conditions, such as operation procedure that we use for observation experiment of the mesentery venule using intravital microscope. Our findings suggested the involvement of mast cells in the initial stage of trauma-induced leukocyte rolling. ${ }^{3,4}$

In this paper, we report a newly devised migration measurement using the histological approach method. We also analyzed and compared TNF $\alpha-$ induced leukocyte migration and IL- $1 \alpha$-induced leukocyte migration. Furthermore, we analyzed casein inflammation, which is a normal form of inflammation characterized by polymorphonuclear leukocyte migration.

\section{Materials and methods}

\section{Agents}

Human recombinant TNF- $\alpha$ and IL- $1 \alpha$ were kindly supplied by Dainippon Pharmaceutical 
Co. LTD, Osaka, Japan. TCV-309 (3-bromo-5-[Nphenyl-N-[2-[[2-(1,2,3,4-tetrahydro-2-isoquinolylcarbonyloxy)ethyl]carbamoyl]ethyl]carbamoyl]-1-propylpyidiniumnitrate) was kindly supplied by Takeda Pharmaceutical Co. LTD, Osaka, Japan. Fucoidin was purchased from Sigma Chemical Co., St. Louis, MO, USA. Giemsa stain was obtained from Merck, Darmstadt, Germany. Casein and Canada balsam were from Wako Pure Chemical Co., Osaka, Japan.

\section{Animals}

Adult male Sprague Dawley (SD) strain rats (200-250 g, Specific-pathogen free, Japan SLC Co. LTD., Hamamatsu, Japan) were used as the source of mesentery tissue samples. These animals were maintained and handled according to the recommendations of experimental animal committee of National Food Research Institute and Kitasato University.

\section{Intraperitoneal challenge with $\mathrm{TNF} \alpha, \mathrm{IL}-1 \alpha$, or Casein}

SD rats, lightly anaesthetized with ethyl ether, were challenged i.p. with TNF $\alpha$ or IL-1 $\alpha$ dissolved in $5 \mathrm{ml}$ of sterile saline warmed to $37^{\circ} \mathrm{C}$, or casein dissolved in $5 \mathrm{ml}$ of Krebs-Ringer Bicarbonate solution warmed to $37^{\circ} \mathrm{C}$. At indicated time points after challenge, the animals were sacrificed by intracardiac injection of $0.2 \mathrm{ml}$ of undiluted pentobarbital (Nembutal ${ }^{\circledR}$ sodium solution, Abbot Laboratories, North Chicago, IL 60064, USA, $60 \mathrm{mg} / \mathrm{ml}$ ). The subsequent rapid circulatory arrest was confirmed by palpation prior to laparotomy for tissue sampling. Systemic treatment with various drugs was performed as described in the results section. At the time of tissue sampling, arterial blood samples were collected from a tail artery for use in determining systemic polymorphonuclear and mononuclear leukocyte (PMNL and MNL) counts. These cell counts were obtained using a Bürker chamber after staining (1:10) with Türk's solution (Wako Pure Chemical Co., Osaka, Japan).

\section{Sampling of mesenteric tissue, histological procedures, and measurements of intravascular and tissue leukocytes}

Samples of intact mesenteric microvascular networks were harvested, fixed in paraformaldehyde (2\%) and glutaraldehyde (5\%), stained with Giemsa stain (1:20), dried, and mounted on glass slides with Canada balsam as described in detail in our previous paper. ${ }^{3}$ Also, the concentrations of $\mathrm{PMN}$ and $\mathrm{MN}$ leukocytes in small venules (inner diameter 20-50 $\mu \mathrm{m}$ ) were calculated assuming cylindrical vessel geometry as described in our previous paper. $^{3}$ Namely rolling leukocyte count at venule means total leukocyte counts minus systemic leukocyte counts.
Therefore, the venular leukocyte content was expressed either as the number of leukocytes per nL of vessel volume or, more commonly, as the ratio of venular leukocyte concentration to systemic leukocyte concentration in order to normalize variations in the systemic leukocyte count (see reference 3, and results section). Mean values of intravascular leukocyte concentrations for the different groups of animals were calculated as the mean for 2-6 vessels per animal. An extravascular leukocyte count was performed within a perivascular area of small venules (inner diameter $20-50 \mu \mathrm{m})$. Within a square area (200 $\mu \mathrm{m} \times 200 \mu \mathrm{m})$ surrounding the venules, infiltrated PMN leukocytes were counted under microscope at a magnification of 1000 times.

\section{Statistics}

For statistical analysis, one-way ANOVA and Dunnet multiple tests for 3 or more groups, or Student's $t$ test for 2 groups were used. $\mathrm{P}$ values $<0.05$ were considered significant. All values are expressed as mean \pm standard error. $\mathrm{N}$ represents the number of animals per group.

\section{Results}

\section{Relationship between venule diameter and PMN migration}

The relationship between venule diameter and PMN migration was obtained on the mesentery preparation in order to decide which area has to be counted for proper measurement. In various sites surrounding the venules of the mesentery, which were $20-50 \mu \mathrm{m}$ in diameter, PMNs were counted to clarify the relation between vessel diameter and migration of PMNs. The formula of relation curve was $y=1.13 \mathrm{x}+$ $104.59 R^{2}=0.0076(n=15, x$ : Diameter of venule, $y$ : $\mathrm{PMN}$ migrated). The results indicated that there is no relation between the diameter of the venule and PMN migration. Therefore, we randomly selected perivascular sites $(20-50 \mu \mathrm{m})$, in which we counted PMNs, in order to produce the migration index.

\section{Time changes in migration of PMNs in inflammation induced by TNF $\alpha, \mathrm{IL}-1 \alpha$, and casein}

Using this method, PMN migration was measured in TNF $\alpha$, IL- $1 \alpha$, and casein-induced inflammation (Fig. 1A). In $\mathrm{TNF} \alpha$-induced inflammation, peak migration is at $3 \mathrm{~h}$ after injection. In IL- $1 \alpha$-induced inflammation, the peak of PMN migration was also at $3 \mathrm{~h}$. In casein-induced inflammation, the first peak in PMN migration was at $2 \mathrm{~h}$ after injection. After that, there was an increase in PMN migration at $5 \mathrm{~h}$. 

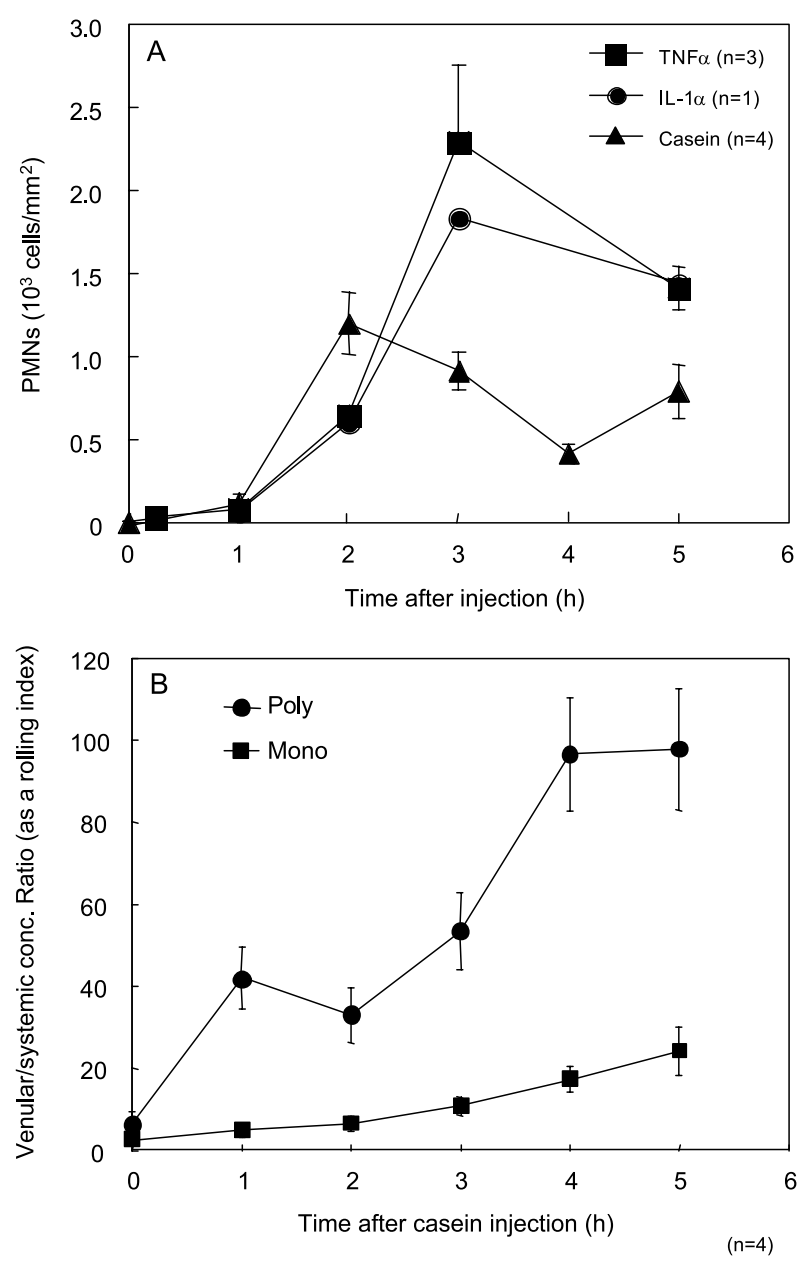

FIG. 1. Time changes of PMNs Migration induced by TNF $\alpha$, $\mathrm{IL}-1 \alpha$, and $1 \%$ casein in mesentary of rats $(A)$, and time changes of venular/systemic concentration ratio (as rolling index) of PMNs and MNs induced by $1 \%$ casein in rats (B). Values were expressed by means \pm standard error.

\section{Time change in PMN and MN leukocyte rolling} in casein-induced inflammation

In casein inflammation, the venular/systemic leukocyte concentration ratio was measured as an index of rolling (Fig. 1B). In PMNs, the first peak was $1 \mathrm{~h}$ after the casein injection and a second peak was observed at $4 \mathrm{~h}$ after the injection. On the other hand, the rolling of MNs increased gradually to peak $5 \mathrm{~h}$ after the casein injection.

\section{Effects of a PAF antagonist and an inhibitor of} selectin on venular/systemic leukocyte concentration ratio (as a rolling index) in casein inflammation at $1 \mathrm{~h}$

In casein inflammation at $1 \mathrm{~h}$, effects of the specific PAF antagonist, TCV $309(0.1 \mathrm{mg} / \mathrm{kg})$ on leukocyte rolling were investigated (Fig. 2A). PAF antagonist TCV 309 and the selectin inhibitor fucoidin were injected intravenously into a tail vein at the same time as a casein injection. TCV 309 had no effect on $1 \mathrm{~h}$ PMN rolling, and likewise, no effect on $1 \mathrm{~h}$ MN rolling. On the other hand, the selectin inhibitor fucoidin had an inhibitory effect on $1 \mathrm{~h} \mathrm{PMN}$ rolling. However it did not affect MN rolling.

\section{Effects of an inhibitor of selectin and a PAF antagonist on PMN migration in $\mathrm{TNF} \alpha, \mathrm{IL}-1 \alpha$, and casein-induced inflammation at 2 and $3 \mathrm{~h}$}

Effects of the selectin inhibitor and PAF antagonist on PMN migration were investigated in TNF $\alpha$, IL- $1 \alpha$, and casein-induced inflammation (Fig. 2B, 2C, and 2D). In TNF $\alpha$ inflammation at $3 \mathrm{~h}$ after injection, fucoidin inhibited PMN migration in a dose-dependent manner. TCV $(0.1 \mathrm{mg} / \mathrm{kg})$ also inhibited migration. In IL$1 \alpha$ inflammation, fucoidin $(10 \mathrm{mg} / \mathrm{kg})$ inhibited PMN migration completely. TCV $(0.1 \mathrm{mg} / \mathrm{kg})$ also inhibited the migration completely (Fig. 2C). In casein inflammation at $2 \mathrm{~h}$ after injection, fucoidin $(1-10 \mathrm{mg} / \mathrm{kg})$ inhibited PMN migration in a dose-dependent manner. TCV $(0.1 \mathrm{mg} / \mathrm{kg})$ also partially inhibited the migration (Fig. 2D)

\section{Discussion}

There are many measurement methods for PMN migration, the most popular in vitro method is the Boyden chamber approach. It is also possible to count migrated PMNs in the inflammatory site, such as peritoneal cavity, or pleural cavity inflammatory pouch in vivo. In histological samples, measurement of myeloperoxidase activity of migrated PMNs in the tissue is often performed instead of counting them. In mesenteric inflammation, this is very useful for rolling measurements because it is easy to detect the venules and to observe the flow of cells in the venules. We have designed a new histological method for rolling measurement. $^{3-5}$ This method is very useful for observation of leukocyte rolling in undisturbed microcirculation. Therefore, we applied it to PMN migration measurement. The method is very convenient for relating rolling and migration because it makes it possible to measure both events in the same sample.

Using this method, it has become clear that the time-lag between PMN rolling and migration is about $1 \mathrm{~h}$. Namely, it takes about $1 \mathrm{~h}$ to migrate from the rolling situation. The histological method has many advantages. One of them is that it is the undisturbed method without surgery-induced trauma in local site. It is very interesting that it is possible to see pure reaction of stimulants in vivo using the method.

In Fig. 1B, Poly means mainly PMN rolling in mesentery venule and Mono means monocyte and lymphocyte rolling. Rolling of PMN increased in the acute inflammation $(0-5 \mathrm{~h})$. On the other hand, rolling of mononuclear leukocytes increased gradu- 

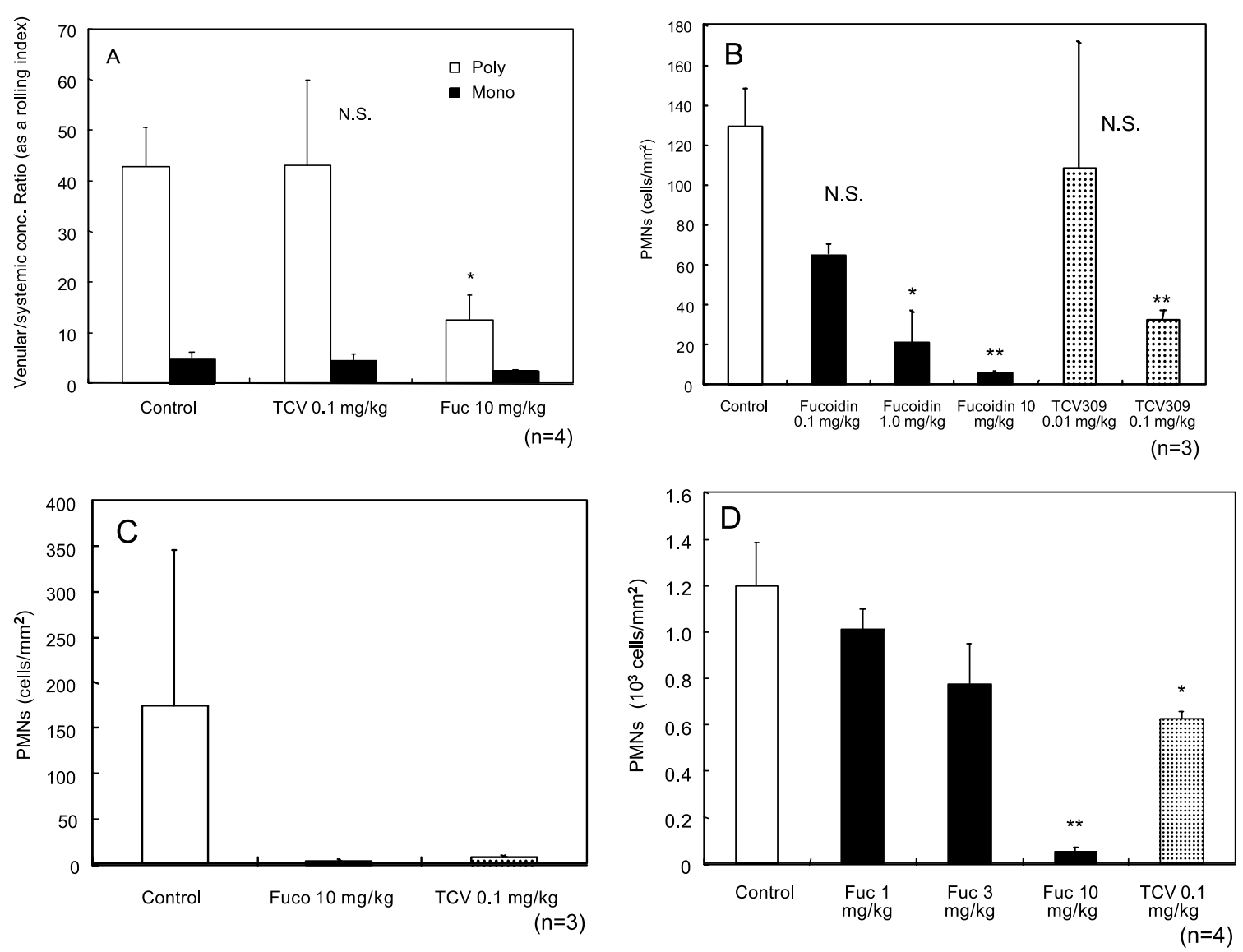

FIG. 2. Effects of PAF antagonist and selectin inhibitor on venular/systemic concentration ratio (as a rolling index) casein induced inflammation in peritoneal cavity (A) and PMNs Migration in peritoneal inflammation of rats induced by TNF $\alpha$ (B), IL$1 \alpha(C)$, and $1 \%$ casein (D). Effects of PAF antagonist and selectin inhibitor on venular/systemic concentration ratio (as rolling index) in peritoneal inflammation induced by $1 \%$ casein were observed at $1 \mathrm{~h}$ after injection (A). PMN Migration induced TNF $\alpha$ and IL- $1 \alpha$ were observed at $3 \mathrm{~h}$ injection. PMN Migration induced $1 \%$ casein was observed at $2 \mathrm{~h}$ injection. Values were expressed by means \pm standard error. N.S. means no significant against control group. ${ }^{*}: p<0.05$ vs Control group. **: $p<0.01$ vs Control group.

ally. In generally, firstly PMNs were recruited to inflammatory site, and secondly mononuclear leukocytes were recruited, finally lymphocytes migrate at the inflammatory site. Therefore it seems that the rolling change of Mono mainly rolling of mononuclear leukocytes. There were no relation between Poly and Mono rolling in acute casein inflammation. It was clear that these cells did independently do rolling on the mesentery venule.

It is thought that PAF or IL-8 activate leukocyte during rolling or firm adhesion, and attract them to the extravascular area. ${ }^{6}$ Therefore, they are very important for extravascular migration of leukocytes. There are some reports about this involvement of PAF in leukocyte (especially eosinophil) migration..$^{7-9}$ However these are mostly culture cell experiments using human umbilical vascular endothelial cells and leukocytes in vitro. We elucidated the PAF action on migration of PMNs in casein inflammation using the selective PAF antagonist, TCV-309. TCV 309 is a selective PAF antagonist, which is very useful for revealing PAF involvement in vivo and in vitro. Some papers have suggested that PAF is particularly in- volved in eosinophil migration. ${ }^{7,8}$ Another paper states that it is involved in leukocyte migration.? Fucoidin is a potent inhibitor of the selectin-induced reaction in vessels, especially rolling and adhesion on the venule wall. ${ }^{10}$ [R1]Sometimes fucoidin is used to elucidate rolling. The results from fucoidin experiments confirm that PMN rolling must occur in the venule wall in order to produce PMN migration

PAF can sometimes activate PMN migration, $\mathrm{H}_{2} \mathrm{O}_{2}$ production, and activate lung tissue to release arachidonic acid products. ${ }^{11,12}$ These reports indicate that it is a leukocyte activator. However from results of inhibitory experiments (Fig. 2), TCV309 did not affect PMN rolling. It is therefore suggested that PAF is not involved in PMN rolling. One report claims that $\mathrm{PAF}$ is involved in leukocyte adhesion. ${ }^{13}$ That is to say, PAF increases shear rate-dependent leukocyte adhesion. However, there are no reports suggesting direct dependence on PAF-induced rolling on leukocyte migration.

TNF $\alpha$ induced PMN migration was partially inhibited by administration of TCV-309 $(0.1 \mathrm{mg} / \mathrm{kg})$. On the other hand, IL- $1 \alpha$-induced PMN migration was 
almost completely inhibited by administration of TCV-309. These results suggest that TNF $\alpha$-induced PMN migration involves not only PAF but also other factors. They also suggest that PAF is very important in IL- $1 \alpha$-induced PMN migration. It is well known that TNF $\alpha$ rapidly induces ICAM-1 on endothelial cell surfaces or eosinophil cell surfaces which is required for leukocyte migration. ${ }^{14,15}$ Young et al reported on the divergent action mechanism between TNF $\alpha$ and IL-1 $\beta$ in leukocyte transmigration. ${ }^{16}$ From these reports, it can be speculated that the mechanisms of these cytokines are different though they have the same effect on leukocyte migration.

These compounds (PAF antagonist and selectin inhibitor) were used for surveying involvement of $\mathrm{PAF}$ and selectin against PMN migration. The results suggest that PAF and selectin involvements are necessary for PMN migration at the mesentery area. In TNF $\alpha$-induced mesentery inflammation, PAF partially involves in PMN migration. On the other hand, PAF-induced PMN migration is necessary for PMN extravasation after rolling. Whereas in casein-induced mesentery inflammation PAF partially involved in PMN migration, it seems similar to TNF-induced mesentery inflammation. From the results, it is suggest that casein induce mesentery inflammation via $\mathrm{TNF} \alpha$ production.

In casein inflammation, PMN migration was shown to be of the TNF $\alpha$ type as opposed to the IL- $1 \alpha$ type, by the fact that the PAF antagonist, TCV, had only a partial effect on PMN migration. Antagonist experiments in casein inflammation show that PAF and other factors, such as IL-8, are involved in PMN migration in the mesentery.

The improved undisturbed microcirculation method is very useful for not only rolling analysis but also analysis of leukocyte migration. Casein inflammation was analyzed using this method, revealing that rolling is necessary and also suggesting that partial involvement of PAF is necessary for pathogenesis of leukocyte extravasation.

ACKNOWLEDGEMENTS. The some parts of experiments were mainly performed at Kitasato University of Pharmaceutical Sciences. We are very appreciative of Ms M. Higashino, N. Nezasa, and Dr. N Kato for their excellent technical assistance as graduate students of Kitasato University.

\section{References}

1. Carlos TM, Harlan JM. Leukocyte-endothelial adhesion molecules. Blood 1994; 84: $2068-2101$

2. Lasky LA. Selectins: interpreters of cell-specific carbohydrate information during inflammation. Science 1992; 258: 964-969.

3. Yamaki K, Lindbom L, Thorlacius H, Hedqvist P, Raud J. An approach for studies of mediator induced leukocyte rolling in the undisturbed microcirculation of the rat mesentery. Br J Pharmacol 1998; 123 $381-389$.

4. Yamaki K, Thorlacius $\mathrm{H}$, Xie X, Lindbom L, Hedqvist P, Raud J. Characteristics of histamine induced leukocyte rolling in the undisturbed microcirculation of the rat mesentery. Br J Pharmacol 1998; 123: 390399.

5. Yamaki K, Takano-Ishikawa Y, Kobori M. Analysis of increased leukocyte ratio induced by tumor necrosis factor- $\alpha$ using several inhibitors in the undisturbed microcirculation of the rat mesentery. International Immunopharmacol 2001; 1: 561-567.

6. Jung U, Norman KE, Scharffetter-Kochanek K, Beaudet AL, Ley K. Transit time of leukocytes rolling through venules controls cytokineinduced inflammatory cell recruitment in vivo. J Clin Invest 1998; 102: $1526-1533$

7. Wardlaw AJ, Moqbel R, Cromwell O, Kay AB. Platelet-activating factor. A potent chemotactic and chemokinetic factor for human eosinophils. $J$ Clin Invest 1986; 78: 1701-1706.

8. Liu L, Zuurbier AE, Mul FP, Verhoeven AJ, Lutter R, Knol EF, Roos D. Triple role of platelet-activating factor in eosinophil migration across monolayers of lung epithelial cells: eosinophil chemoattractant and priming agent and epithelial cell activator. J Immunol 1998; 161: $3064-$ 3070

9. Cepinskas G, Sandig M, Kvietys PR. PAF-induced elastase-dependent neutrophil transendothelial migration is associated with the mobilization of elastase to the neutrophil surface and localization to the migrating front. J Cell Sci 1999; 112: 1937-1945.

10. Lindbom L, Xie X, Raud J, Hedqvist P. Chemoattractant-induced firm adhesion of leukocytes to vascular endothelium in vivo is critically dependent on initial leukocyte rolling. Acta Physiol Scand 1992; 146: $415-421$

11. Gastaldello K, Husson C, Wens R, Vanherweghem JL, Tielemans. Role of complement and platelet-activating factor in the stimulation of phagocytosis and reactive oxygen species production during haemodialysis. Nephrol Dial Transplant 2000; 15: 1638-1646.

12. Grimminger $\mathrm{F}$, Mayer $\mathrm{K}$, Kiss L, Walmrath $\mathrm{D}$, Seeger W. PAF-induced synthesis of tetraenoic and pentaenoic leukotrienes in the isolated rabbit lung. Am J Physiol Lung Cell Mol Physiol 2000; 278: L268-L275.

13. Bienvenu K, Russell J, Granger DN. Platelet-activating factor promotes shear rate-dependent leukocyte adhesion in postcapillary venules. $J$ Lipid Mediat 1993; 8: 95-103.

14. Javaid K, Rahman A, Anwar KN, Frey RS, Minshall RD, Malik AB. Tumor necrosis factor-alpha induces early-onset endothelial adhesivity by protein kinase Czeta-dependent activation of intercellular adhesion molecule-1. Circ Res 2003; 92: 1089-1097.

15. Ip WK, Wong CK, Lam CW. Tumour necrosis factor-alpha-induced expression of intercellular adhesion molecule-1 on human eosinophilic leukaemia EoL-1 cells is mediated by the activation of nuclear factorkappaB pathway. Clin Exp Allergy 2003; 33: 241-248.

16. Young RE, Thompson RD, Nourshargh S. Divergent mechanisms of action of the inflammatory cytokines interleukin 1-beta and tumour necrosis factor-alpha in mouse cremasteric venules. $\mathrm{Br} J$ Pharmacol 2002; 137: 1237-1246.

\section{Received 9 September 2003 \\ Accepted 4 November 2003}




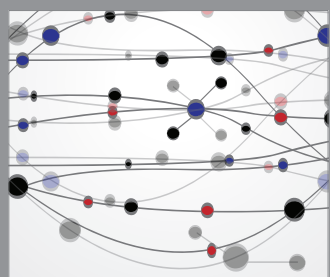

The Scientific World Journal
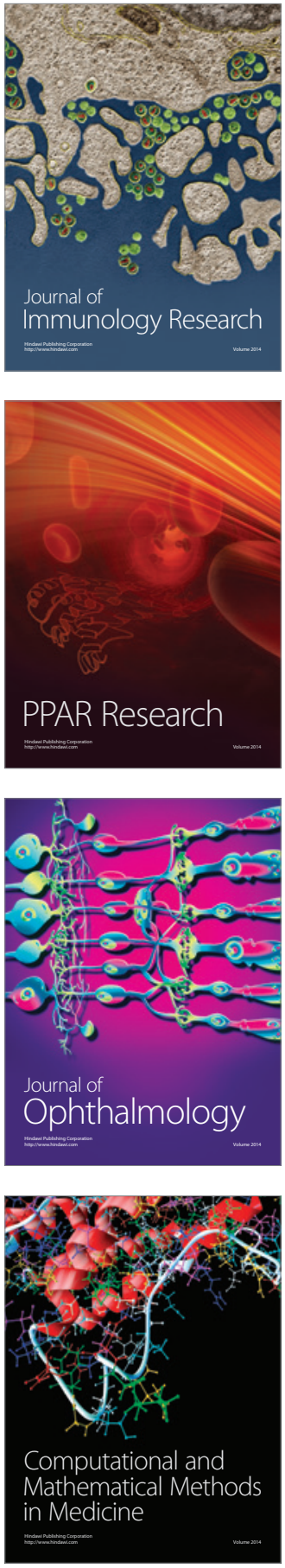

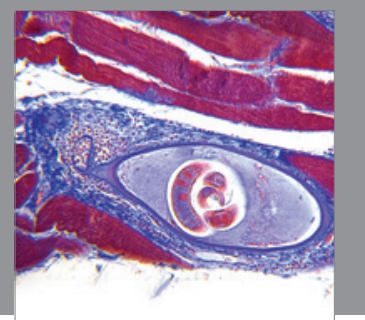

Gastroenterology

Research and Practice
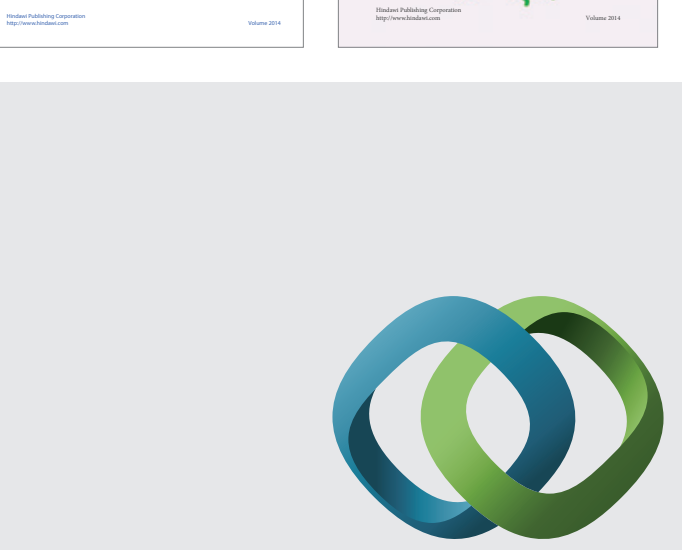

\section{Hindawi}

Submit your manuscripts at

http://www.hindawi.com
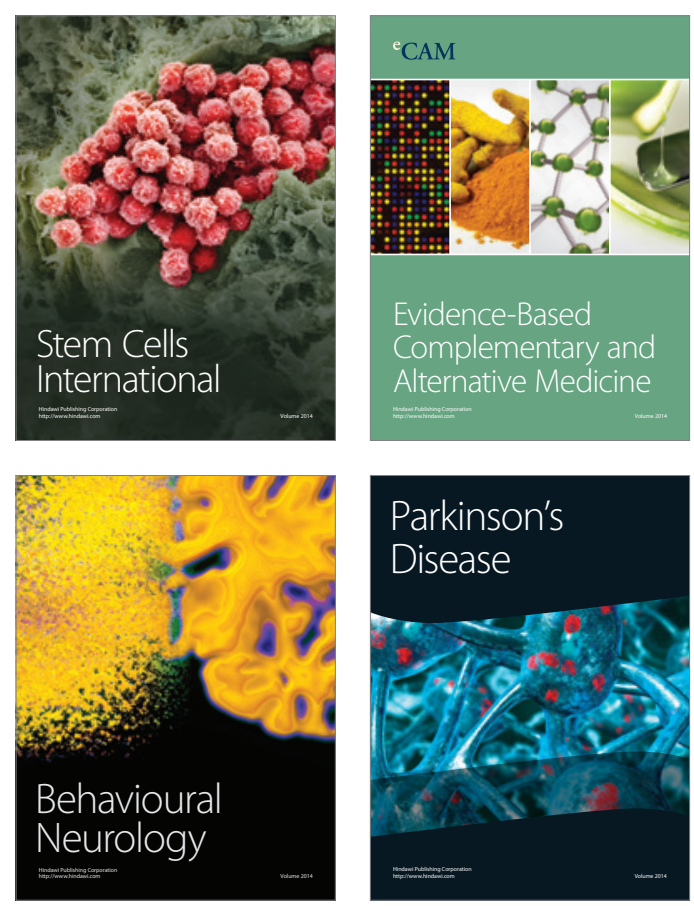

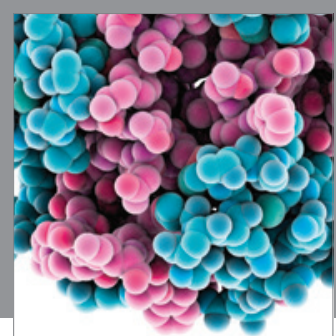

Journal of
Diabetes Research

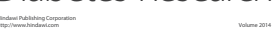

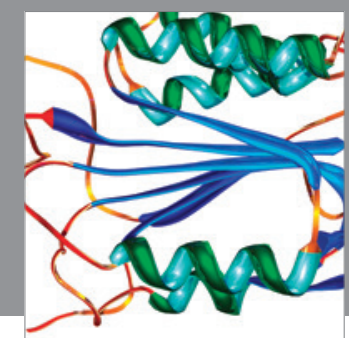

Disease Markers
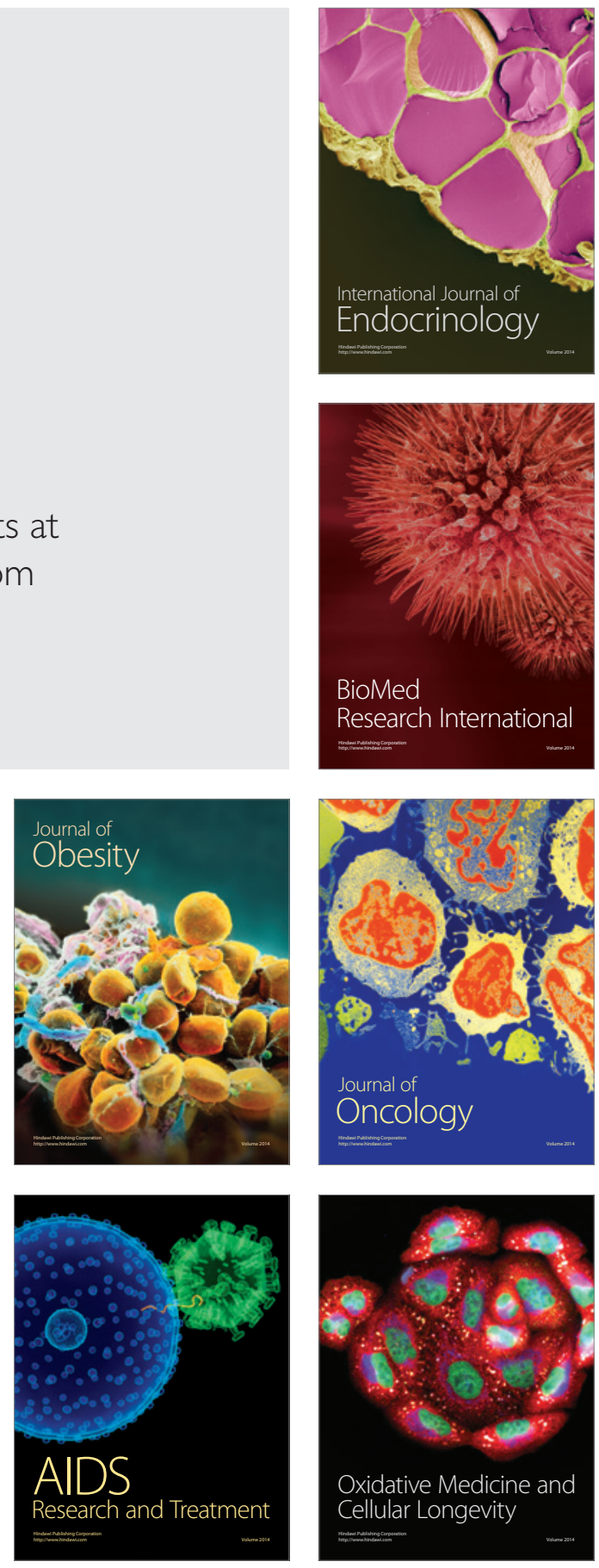